\title{
Cobertura do exame citopatológico do colo do útero no Estado de Minas Gerais, Brasil, no período entre 2000-2010: um estudo a partir dos dados do Sistema de Informação do Câncer do Colo do Útero (SISCOLO)
}

\author{
Cervical cancer screening coverage in the state of \\ Minas Gerais, Brazil between 2000-2010: a study using data from the \\ Cervical Cancer Information System (SISCOLO)
}

\author{
Gabriel Winston de Carvalho Nascimento', Claudia Cristina de Aguiar Pereira², \\ David Israel de Carvalho Nascimento ${ }^{1}$, Galileu Chagas Lourenço', \\ Carla Jorge Machado ${ }^{3}$
}

Resumo

O estudo analisou o rastreamento do câncer do colo do útero em Minas Gerais, Brasil, quanto ao número de exames realizados anualmente e à cobertura do exame citopatológico. A fonte de dados foi o Sistema de Informação do Câncer do Colo do Útero (SISCOLO). Utilizou-se um estudo de série temporal referente à realização do exame, no período entre 2000-2010, em mulheres de 25 a 59 anos, residentes nesse Estado e usuárias do Sistema Único de Saúde (SUS). Realizaram-se comparações em relação às metas pactuadas para o Programa Viva Mulher para as macrorregiões. Observou-se cobertura inferior à meta pactuada pelo Programa Viva Mulher (63,8\%) e à estimada pelos critérios populacionais $(66,4 \%)$. Em 2000, as macrorregiões que apresentaram maior cobertura em relação às duas metas foram: Triângulo do Norte, Triângulo do Sul e Centro. No ano de 2010, observaram-se mudanças em relação à posição ocupada pelas macrorregiões e melhorias na cobertura. Quanto à meta SUS, as macrorregiões Nordeste, Jequitinhonha e Sul apresentaram as maiores coberturas. As diferenças entre as duas metas avaliadas neste estudo sobre a cobertura da realização do exame de Papanicolau destacam a importância da escolha do critério para estabelecimento de metas.

Palavras-chave: rastreamento; câncer do colo do útero; prevenção; cobertura.

\footnotetext{
Abstract

This study aimed to analyze the cervical cancer screening in Minas Gerais, Brazil, regarding the number of tests performed annually and its coverage. We used data from the Cervical Cancer Information System (SISCOLO). Screenings performed between 2000 and 2010 were analyzed regards time sequence for women who lived in the state, aged 25-59 and users of the Unified Health System (SUS). We performed comparisons against target goals agreed for the Viva Mulher Program in the macroregions. The overall

${ }^{1}$ Acadêmico de Medicina na Faculdade de Medicina da Universidade Federal de Minas Gerais (UFMG) - Belo Horizonte (MG), Brasil.

${ }^{2}$ Pesquisadora em Saúde Pública da Escola Nacional de Saúde Pública Sergio Arouca (ENSP/Fiocruz) - Rio de Janeiro (RJ), Brasil.

${ }_{3}^{3}$ Professor Associado II do Departamento de Medicina Preventiva da Faculdade de Medicina da Universidade Federal de Minas Gerais (UFMG) - Belo Horizonte (MG), Brasil.

Trabalho realizado na Universidade Federal de Minas Gerais (UFMG) e Escola Nacional de Saúde Pública Sergio Arouca (ENSP/Fiocruz) - Belo Horizonte (MG) e Rio de Janeiro (RJ), Brasil. Endereço para correspondência: Claudia Cristina de Aguiar Pereira - Rua Leopoldo Bulhões, 1480, $7^{0}$ andar - CEP: 21041-210 - Rio de Janeiro (RJ), Brasil E-mail: cpereira@ensp.fiocruz.br; pereirac.claudia@gmail.com

Fonte de financiamento: nenhuma.

Conflito de interesses: nada a declarar
} 
coverage was lower than the one set by Viva Mulher Program (63.8\%) and also lower than the estimated by the population criteria (66.4\%). In 2000, macroregions that showed the highest coverage for the two goals were: Northern Triangle, South and Central Triangle. In 2010, there were changes regarding the position occupied by the macroregions and coverage improvements. As for SUS targets, the Northern, Jequitinhonha and South macro-regions had the highest coverage. The differences between the two targets evaluated in this study highlight the importance of the criteria chosen for setting goals.

Keywords: screening; cervical cancer; prevention; coverage.

\section{INTRODUÇÃO}

O câncer do colo do útero (CCU) é o terceiro tipo de câncer mais comum entre as mulheres, com aproximadamente $530 \mathrm{mil}$ novos casos por ano no mundo, e o responsável pelo óbito de 270 mil mulheres por ano ${ }^{1}$. No Brasil, em 2014, foram estimados 15.590 novos casos $^{2}$. Trata-se de uma doença rara em mulheres com até 30 anos e sua incidência aumenta progressivamente até ter seu pico na faixa de 40 a 50 anos. A mortalidade aumenta, de modo progressivo, a partir da quarta e quinta década de vida, com expressivas diferenças regionais no país. A incidência de CCU é aproximadamente o dobro em países em desenvolvimento, comparativamente aos países desenvolvidos, e trata-se de uma doença relacionada ao baixo nível socioeconômico da população $0^{1,3,4}$.

Por ser uma neoplasia causada pelo Papilomavírus humano (HPV), a realização do exame de Papanicolau, também conhecido como exame citopatológico do colo do útero, exame colpocitopatológico ou oncocitológico, tem sido reconhecida mundialmente como uma estratégia segura e eficiente para a detecção precoce do CCU na população feminina. A efetividade da detecção precoce de lesões precursoras por meio do exame de Papanicolau, associada ao tratamento em seus estádios iniciais, tem resultado em redução de até $90,0 \%$ nas taxas de incidência de câncer cervical invasor, quando o rastreamento apresenta boa cobertura e é realizado dentro dos padrões de qualidade ${ }^{5}$. O Ministério da Saúde recomenda o rastreamento do CCU a partir dos 25 anos de idade para as mulheres sexualmente ativas. O intervalo deve ser de três anos entre os exames, quando ocorrem dois exames negativos com intervalo anual. A recomendação atual preconiza a continuidade dos exames até os 64 anos de idade, devendo ser interrompidos quando há pelo menos dois exames negativos nos últimos cinco anos ${ }^{6}$. A idade mínima para interrupção do rastreamento em recomendações anteriores (antes de 2011) era de 59 anos $^{7}$.

Em 1995, como parte de um compromisso assumido pelo Brasil na Conferência Mundial sobre a Saúde da Mulher, o Ministério da Saúde elaborou um projeto-piloto de rastreamento do CCU no país denominado "Viva Mulher: Programa Nacional de Controle do Câncer do Colo do Útero" ${ }^{\text {8-10 }}$. Em 1997, o Programa foi implementado em seis localidades e, no ano seguinte, foi ampliado para todo o território nacional. Também, em 1998, foi criado o Sistema de Informação do Câncer do Colo do Útero (SISCOLO), com o objetivo de monitorar e de gerenciar as ações do Programa, promovendo a análise dos dados coletados nos postos de saúde e nos laboratórios integrados ao Sistema Único de Saúde (SUS). A partir de então, as estratégias de prevenção e de controle do CCU passaram por períodos de reavaliação e de reafirmação - o mais recente foi no ano de 2011, com o lançamento do Programa de Fortalecimento da Rede de Prevenção, Diagnóstico e Tratamento do Câncer do Colo do Útero e de $\mathrm{Mama}^{6}$. Metas quanto ao número de exames a serem realizados foram pactuadas ${ }^{11}$. Diante da magnitude do CCU e da importância de sua prevenção por rastreamento ${ }^{12}$, é relevante quantificar as informações sobre a sua prevenção para a elaboração de políticas públicas. Para tanto, o número de exames preventivos deve ser observado à luz das metas pactuadas e preconizadas pelo SUS. Essas mesmas metas devem ser observadas e consideradas a partir de informações populacionais para que o dimensionamento seja o mais adequado possível, segundo as mudanças demográficas na população-alvo.

Assim, os objetivos deste estudo foram descrever a série temporal de informações sobre os exames de Papanicolau realizados pelo SUS em Minas Gerais, localizado na região Sudeste do Brasil, entre 2000 e $2010^{13}$. Apesar da queda da mortalidade por CCU nesse Estado, a cobertura do rastreamento foi ressaltada como insatisfatória ${ }^{13}$. Minas Gerais, além de ter o maior número de municípios do país, possui ainda grande heterogeneidade socioeconômica entre suas macrorregiões. Essas constatações tornam propícia a observação de uma série temporal no período após a implementação do SISCOLO em Minas Gerais, o que torna possível analisar quando houve intensificação das iniciativas voltadas para o CCU, principalmente no que tange à sua prevenção.

\section{MATERIAIS E MÉTODOS}

Trata-se de estudo ecológico de série temporal que avaliou informações epidemiológicas sobre a realização do exame de Papanicolau em Minas Gerais no período 2000 a 2010. Os dados de população utilizados provieram do sistema informatizado SISCOLO ${ }^{10}$, especificamente desenvolvido pelo Departamento de Informática do Sistema Único de Saúde, em parceria com o Instituto Nacional do Câncer (INCA), e voltado para a estruturação do Programa Viva Mulher ${ }^{11}$, que coleta e processa informações sobre identificação de pacientes e laudos de exames cito e histopatológicos, fornecendo dados para o monitoramento 
externo da qualidade dos exames. Os dados foram extraídos diretamente do sítio do DATASUS.

Foram utilizadas informações sobre as mulheres residentes no Estado de Minas Gerais, com idade de 25 a 59 anos $^{14}$, e estimativas do número de usuárias do SUS. Para tal, foi necessário subtrair das informações de cada município o número de mulheres beneficiárias de planos privados de saúde (saúde suplementar) ${ }^{15}$. O cálculo realizado foi referente ao mês de junho de cada ano estudado, fazendo a subtração a partir do total de mulheres de cada município. Obteve-se, assim, a estimativa do número de mulheres atendidas pelo SUS em cada ano. A premissa básica foi que o exame citopatológico deveria ser realizado uma vez ao ano, em mulheres de 25 a 59 anos de idade ${ }^{7,16}$, ou que já tivessem tido atividade sexual mesmo antes dessa faixa etária, e depois de dois exames anuais consecutivos negativos, a cada três anos, conforme preconizado pelo Ministério da Saúde ${ }^{17}$. Assim, para este trabalho, definiu-se que o número de mulheres atendidas deveria ser no mínimo igual a um terço da população de usuárias do SUS (não beneficiária de saúde suplementar) - definido como o parâmetro mínimo do quantitativo de exames. Além disso, para comparação, obtivemos as metas pactuadas para o Programa Viva Mulher de cada município, por meio de dados disponíveis no SISCOLO, as quais foram definidas de acordo com a população-alvo para o rastreamento ( 25 a 59 anos), com base na população anual de mulheres estimada pelo Instituto Brasileiro de Geografia e Estatística (IBGE). Para 2009, a meta SUS (para todo o Estado) equivaleu a 20,0\% da população-alvo; para 2010, o indicador equivaleu a $21,0 \%$; para os demais anos, sabe-se que não superou 30,0\%, conforme preconizado pelo Ministério da Saúde ${ }^{18}$, mas não se tem o conhecimento exatamente do fator utilizado na multiplicação.

\section{Análise estatística}

Os dados quantitativos foram recebidos em planilhas do Microsoft Excel 2010. As frequências absolutas e os valores calculados quanto às coberturas foram representados por tabelas e gráficos. Após conversão dos dados em formato compatível com pacote estatístico STATA, versão $13.1^{19}$, analisaram-se os dados com este último programa.

Foram obtidos, de cada um dos 11 anos estudados - 2000 a 2010 -, os números de exames realizados para as 13 macrorregiões de Minas Gerais e para o total do Estado. Obtiveram-se duas metas para o número de exames ano a ano para cada macrorregião: meta proposta pelo SUS para cada macrorregião (número de exames anuais programados pelo SUS, chamado aqui de meta SUS); meta estimada neste trabalho, considerando-se a população feminina de 25 a 59 anos, residente em cada macrorregião (denominada de meta populacional). De posse dessas informações, estimaram-se as coberturas dessas duas metas. A divisão do número de exames realizados pelo número de exames necessários pela meta SUS vezes 100 resultou na cobertura em relação à meta SUS. Da mesma forma, a cobertura em relação à meta populacional foi obtida pela divisão do número de exames efetivamente realizados pelo número de exames necessários pela meta populacional vezes 100 .

A Figura 1 apresenta as macrorregiões de Minas Gerias definidas pelo Plano de Regionalização do SUS (PDR/SUS) (Figura 1). Os gráficos (Figura 2) indicam a tendência temporal anual entre o período de 2000 e 2010 do número de exames realizados, tanto da meta populacional quanto da meta SUS, para cada uma das macrorregiões.

O estudo obteve aprovação do Comitê de Ética em Pesquisa da Faculdade de Medicina da Universidade Federal de Minas Gerais (UFMG), com parecer n. 510.095, de 14 de janeiro de 2014, e CAAE n. 17983113.6.0000.5149. O Termo de Consentimento Livre e Esclarecido não foi necessário por serem dados secundários.

\section{RESULTADOS}

No ano 2000, o Estado de Minas Gerais teve 1.193.472 exames de Papanicolau programados para a meta SUS e 1.241 .572 para a meta populacional. Contudo, o número de exames efetivamente realizados foi de 792.331, indicando cobertura inferior tanto em relação à meta SUS $(63,8 \%)$ quanto à meta populacional $(66,4 \%)$ (Tabela 1$)$.

As macrorregiões que apresentaram maior cobertura em 2000 em relação à meta SUS foram: Triângulo do Norte, Triângulo do Sul e Centro. No entanto, quando o critério de comparação foi a meta populacional, a macrorregião Centro apresentou maior cobertura que a Triângulo do Sul. As piores coberturas foram observadas em Jequitinhonha, Nordeste e Leste do Norte (meta SUS) e Jequitinhonha, Noroeste e Leste do Norte (meta populacional).

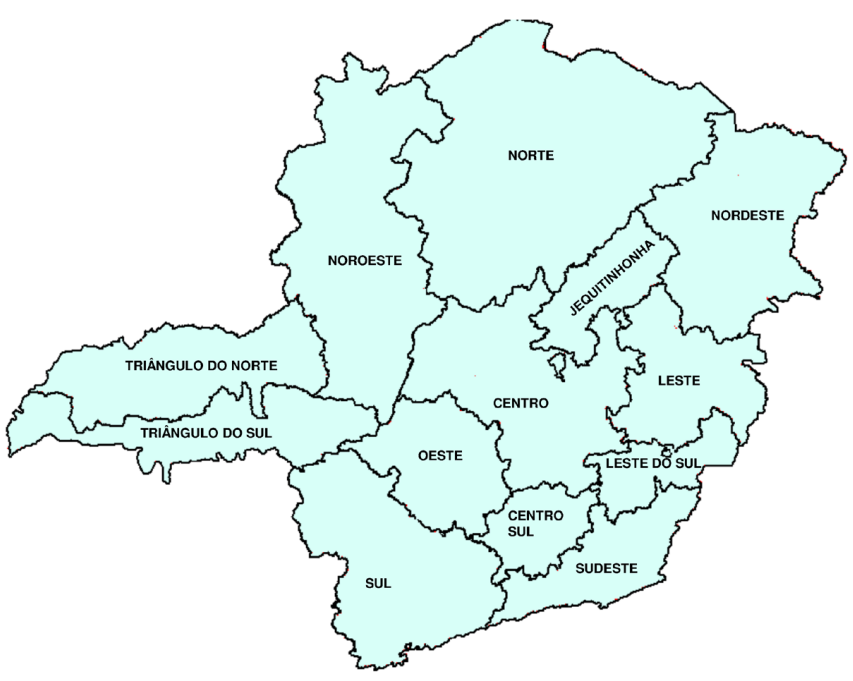

Figura 1. Macrorregiões de saúde de Minas Gerais - Plano de Regionalização do Sistema Único de Saúde (PDR-SUS). Fonte: Secretaria de Estado da Saúde de Minas Gerais (SES-MG)/SMACSS/DEA ${ }^{18}$ 
Em 2010, as metas SUS e populacional foram iguais a 1.495.182 e 1.398.922, respectivamente, com 1.334.940 exames realizados e coberturas iguais a $89,3 \%$ pela meta SUS e a $95,4 \%$ pela meta populacional (Tabela 2). Observou-se, assim, melhoria da cobertura quando comparada à de 2000. No ano de 2010, a posição ocupada pelas macrorregiões mudou comparativamente ao ano de 2000. Quanto à meta SUS, as macrorregiões Nordeste, Jequitinhonha e Sul apresentaram as maiores coberturas.
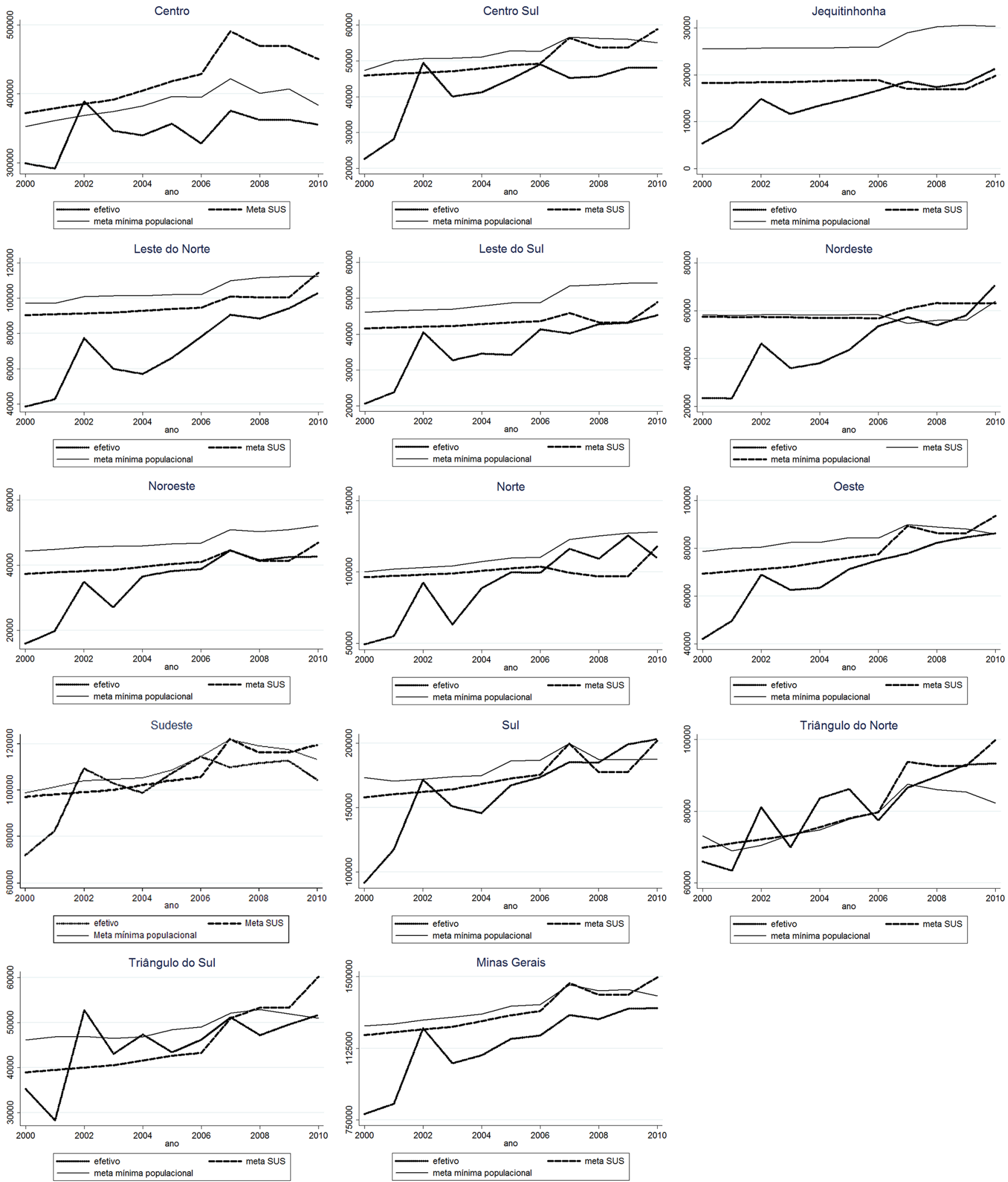

Figura 2. Série temporal do número de exames citopatológicos realizados (efetivo), meta populacional e meta SUS, por macrorregião de Minas Gerais e Estado total (2000-2010) 
Contudo, na meta populacional, Jequitinhonha teve a pior menor cobertura.

Em relação às tendências temporais, nota-se que todas as macrorregiões apresentaram aumento do número efetivo de exames realizados, observando-se um pico em 2002 (Figura 2).
Chama atenção, na macrorregião Centro, o fato de que a meta mínima populacional esteve sempre abaixo da meta SUS, bem como do número de exames realizados, com exceção do ano de 2002. A macrorregião Triângulo do Norte apresentou número de exames realizados acima do estimado pela meta populacional na

Tabela 1. Demonstrativo do número de exames realizados em mulheres de Minas Gerais no Sistema Único de Saúde em 2000, meta populacional estimada, meta SUS e cobertura em relação às metas

\begin{tabular}{|c|c|c|c|c|c|}
\hline Macrorregiões & $\begin{array}{c}\text { Número de exames } \\
\text { realizados (Efetivo) } \\
\text { Coluna } 1\end{array}$ & $\begin{array}{c}\text { Número mínimo de } \\
\text { exames necessários } \\
\text { (meta populacional) } \\
\text { Coluna } 2\end{array}$ & $\begin{array}{c}\text { Meta proposta } \\
\text { (número de exames } \\
\text { programados - meta } \\
\text { SUS) } \\
\text { Coluna } 3\end{array}$ & $\begin{array}{c}\text { Cobertura em } \\
\text { relação à meta } \\
\text { populacional } \\
(\text { Coluna } 1) \times(100) / \\
(\text { Coluna } 2)\end{array}$ & $\begin{array}{c}\text { Cobertura em } \\
\text { relação à meta SUS } \\
(\text { Coluna }) \times(100) / \\
(\text { Coluna } 3)\end{array}$ \\
\hline Sul & 91.580 & 173.336 & 157.812 & 52,8 & 58,0 \\
\hline Centro-Sul & 22.707 & 47.363 & 45.936 & 47,9 & 49,4 \\
\hline Centro & 299.299 & 353.002 & 372.216 & $84,8^{\star *}$ & $80,4^{\star *}$ \\
\hline Jequitinhonha & 5.429 & 25.549 & 18.276 & 21,2 & 29,7 \\
\hline Oeste & 42.143 & 78.720 & 69.408 & 53,5 & 60,7 \\
\hline Leste do Norte & 38.511 & 97.392 & 90.348 & 39,5 & 42,6 \\
\hline Sudeste & 71.893 & 98.772 & 96.936 & $72,8^{\star}$ & $74,2^{*}$ \\
\hline Norte de Minas & 49.358 & 100.103 & 96.456 & 49,3 & 51,2 \\
\hline Noroeste & 15.953 & 44.292 & 37.320 & 36,0 & 42,7 \\
\hline Leste do Sul & 20.670 & 46.154 & 41.640 & 44,8 & 49,6 \\
\hline Nordeste & 23.566 & 57.604 & 58.320 & 40,9 & 40,4 \\
\hline Triângulo do Sul & 35.250 & 46.144 & 39.000 & $76,4^{*}$ & $90,4^{* * *}$ \\
\hline $\begin{array}{c}\text { Triângulo do } \\
\text { Norte }\end{array}$ & 65.972 & 73.141 & 69.804 & $90,2^{\star * *}$ & $94,5^{\star * *}$ \\
\hline TOTAL & 792.331 & 1.241 .572 & 1.193 .472 & 63,8 & 66,4 \\
\hline
\end{tabular}

Fonte: Ministério da Saúde/Departamento de Informática do Sistema Único de Saúde (DATASUS ${ }^{20}$; Instituto Nacional do Câncer (INCA)/Sistema de Informação do Câncer do Colo do Útero (SISCOLO) ${ }^{14}$; Instituto Brasileiro de Geografia e Estatística (IBGE) ${ }^{10}$. ${ }^{*}$ Cobertura acima de $70,0 \%$. ${ }^{* *}$ Cobertura acima de $80,0 \%$. ${ }^{* * *}$ Cobertura acima de $90,0 \%$

Tabela 2. Demonstrativo do número de exames realizados em mulheres de Minas Gerais no Sistema Único de Saúde em 2010, meta populacional estimada, meta SUS e cobertura em relação às metas

\begin{tabular}{|c|c|c|c|c|c|}
\hline Macrorregiões & $\begin{array}{c}\text { Número de exames } \\
\text { realizados (Efetivo) } \\
\text { Coluna } 1\end{array}$ & $\begin{array}{c}\text { Número mínimo de } \\
\text { exames necessários } \\
\text { (meta populacional) } \\
\text { Coluna } 2\end{array}$ & $\begin{array}{c}\text { Meta proposta } \\
\text { (número de exames } \\
\text { programados - meta } \\
\text { SUS) } \\
\text { Coluna } 3\end{array}$ & $\begin{array}{l}\text { Cobertura em } \\
\text { relação à meta } \\
\text { populacional } \\
(\text { Coluna } 1) \times(100) / \\
(\text { Coluna } 2)\end{array}$ & $\begin{array}{c}\text { Cobertura em } \\
\text { relação à meta SUS } \\
(\text { Coluna }) \times(100) / \\
(\text { Coluna } 3)\end{array}$ \\
\hline Sul & 202.971 & 187.552 & 201.660 & $108,2^{\star * *}$ & $100,7^{\star * *}$ \\
\hline Centro-Sul & 48.083 & 55.011 & 58.812 & $87,4^{\star *}$ & $81,8^{\star *}$ \\
\hline Centro & 355.513 & 383.651 & 450.648 & $92,7^{\star * *}$ & $78,9^{*}$ \\
\hline Jequitinhonha & 21.303 & 30.316 & 19.764 & $70,3^{*}$ & $107,8^{\star * *}$ \\
\hline Oeste & 86.271 & 85.941 & 93.468 & $100,4^{\star * *}$ & $92,3^{* * *}$ \\
\hline Leste do Norte & 102.976 & 112.386 & 114.372 & $91,6^{* * *}$ & $90,0^{* * *}$ \\
\hline Sudeste & 104.241 & 113.171 & 119.388 & $92,1^{\star * *}$ & $87,3^{\star *}$ \\
\hline Norte de Minas & 109.823 & 128.061 & 117.960 & $85,8^{\star *}$ & $93,1^{\star * *}$ \\
\hline Noroeste & 42.625 & 52.162 & 46.848 & $81,7^{\star \star}$ & $91,0^{\star * *}$ \\
\hline Leste do Sul & 45.327 & 54.206 & 48.294 & $83,6^{\star *}$ & $93,9^{* * *}$ \\
\hline Nordeste & 70.825 & 63.215 & 64.032 & $112,0^{* * *}$ & $110,6^{* * *}$ \\
\hline Triângulo do Sul & 51.689 & 50.985 & 60.180 & $101,4^{* * *}$ & $85,9^{* *}$ \\
\hline $\begin{array}{c}\text { Triângulo do } \\
\text { Norte }\end{array}$ & 93.293 & 82.265 & 99.756 & $113,4^{\star * *}$ & $93,5^{\star \star \star}$ \\
\hline TOTAL & 1.334 .940 & 1.398 .922 & 1.495 .182 & $95,4^{\star * *}$ & $89,3^{* *}$ \\
\hline
\end{tabular}

Fonte: Ministério da Saúde/Departamento de Informática do Sistema Único de Saúde (DATASUS) ${ }^{20}$; Instituto Nacional do Câncer (INCA)/Sistema de Informação do Câncer do Colo do Útero (SISCOLO) ${ }^{14}$; Instituto Brasileiro de Geografia e Estatística (IBGE) ${ }^{10}$. ${ }^{*}$ Cobertura acima de $70,0 \%$. ${ }^{* *}$ Cobertura acima de $80,0 \%$. ${ }^{* * *}$ Cobertura acima de $90,0 \%$ 
maior parte do período estudado. Contudo, essa macrorregião, a Triângulo do Sul e a Sudeste registraram grandes oscilações no número de exames realizados, principalmente até o ano de 2008. A macrorregião Nordeste teve meta populacional bastante semelhante à meta SUS até 2006, período em que o número de exames realizados permaneceu bem abaixo em ambas. Após 2006, o número efetivo de exames realizados passou a aumentar e se aproximou das metas, até que, no final do período, ultrapassou ambas. A macrorregião Norte também apresentou avanços no número de exames realizados a partir de 2006, quando se aproximou mais das metas. No caso da macrorregião Jequitinhonha, a máxima cobertura obtida foi em relação à meta SUS em 2010; somente após 2008 que o número de exames realizados passou a ser mais elevado que a meta SUS. Seis macrorregiões - Centro-Sul, Leste do Norte, Leste do Sul, Noroeste, Oeste e Sul - apresentaram padrões nítidos, com estimativas pelas metas populacionais mais elevadas que as metas SUS. Estas últimas, por sua vez, permaneceram mais elevadas que o quantitativo realizado na maior parte do período analisado.

Para o Estado como um todo, o número efetivo de exames ficou sempre aquém das metas, independentemente do critério, com exceção de 2002, quando o número realizado se aproximou da meta SUS, mas ficou abaixo da meta populacional.

\section{DISCUSSÃO}

De maneira geral, os resultados indicam que a meta populacional, comparativamente à meta proposta pelo SUS, por meio da Secretaria de Estado da Saúde de Minas Gerais (SES-MG), é diferente em cada macrorregião. Também indicam que houve avanços inequívocos no sentido do aumento da população atendida. A intensificação da política de rastreamento, por intermédio do fortalecimento e da qualificação da rede de atenção básica, e a ampliação dos centros de referência em $2002^{2}$ foram ações que podem ser inferidas com base no aumento da cobertura e no número de exames realizados. Naquele período, priorizaram-se as mulheres entre 35 e 49 anos e que jamais haviam sido submetidas ao exame ou que estavam há mais de três anos sem realizá-lo, alcançando mais de 3,8 milhões de mulheres.

A identificação da cobertura ideal não é tarefa fácil. O cadastro do Sistema de Informações de Atenção Básica (SIAB) é discordante da população anual estimada pelo IBGE e obtida no sítio do DATASUS ${ }^{17}$. Neste trabalho optou-se por efetuar uma estimativa populacional do número mínimo de exames, excluindo-se a população coberta por planos de saúde suplementar (meta populacional). Em seguida, comparou-se a meta populacional à meta anual de realização do exame preconizada pelo Ministério da Saúde (meta SUS). O resultado foi que, em qualquer uma das metas, a cobertura tem aumentado de forma sustentada. Entretanto, os dados evidenciam que uma das macrorregiões mais pobres do Estado, o Jequitinhonha, apresentou grande disparidade entre a meta populacional e a meta SUS. Comparativamente à meta populacional, os números de exames estiveram muito abaixo do esperado ao longo do período de estudo.

Ao analisar os fatores que levaram ao não alcance das metas em relação à cobertura do exame citopatológico, percebe-se que o problema é complexo e multifacetado. Um estudo que abordou o vínculo entre a população em três municípios do Vale do Jequitinhonha ${ }^{21}$ mostrou que os profissionais entrevistados enfatizaram o problema da rotatividade nas Equipes de Saúde da Família, o que interfere negativamente na dinâmica do trabalho e no vínculo construído com as famílias. Esse vínculo é uma ferramenta que possibilita a realização de atos terapêuticos ${ }^{22}$. Dessa forma, pode-se inferir que a rotatividade atuaria como um empecilho no alcance de um maior número de usuárias com exame citopatológico realizado. Outro problema seria a barreira da própria mulher em relação ao exame de Papanicolau. Infelizmente, muitas mulheres, devido ao baixo grau de escolaridade e por residirem em regiões de extrema pobreza, não têm informações adequadas a respeito do câncer cervical nem acerca da sua prevenção e deteç̧ão precoce ${ }^{23}$. Ademais, por ser um exame pélvico, muitas mulheres sentem vergonha de realizá-lo, fazem associação com a dor ou são coibidas por seus parceiros ${ }^{23}$. No entanto, nas sociedades contemporâneas, é considerável o papel das mulheres como pilares de sustento de suas famílias, o que pode levar à negligência nos cuidados com a própria saúde, fazendo com que essas mulheres não procurem os centros de saúde para a realização do exame ${ }^{24}$.

A dificuldade do acesso das usuárias ao SUS para a coleta do exame citopatológico ${ }^{25}$ também pode ser um dos motivos para o não cumprimento das metas de cobertura. Muitas mulheres têm dificuldade em agendar uma consulta com um profissional de saúde ${ }^{25}$. Além disso, a longa distância da unidade básica de saúde, os horários não flexíveis e a falta de estrutura do centro de saúde são alguns dos fatores que dificultam a cobertura do exame do Papanicolau em diferentes regiões ${ }^{26}$. A busca ativa, por meio de sensibilização das mulheres nas comunidades para realização do exame, e a demanda espontânea contribuem para $o$ alcance das metas ${ }^{17}$. Outro aspecto que vai além da realização dos exames preventivos, mas que é de fundamental importância, diz respeito ao treinamento dos profissionais da atenção básica para diagnóstico precoce das lesões precursoras do CCU, o que pode favorecer muito o tratamento e aumentar a possibilidade de sucesso no combate a esse tipo de câncer.

O estudo da cobertura do exame do Papanicolau em Minas Gerais é essencial para a elaboração de estratégias de prevenção, bem como para a avaliação da efetividade das medidas já 
implementadas por meio do Programa Viva Mulher ${ }^{27,28}$. Esse conhecimento é fundamental para ajustes nas políticas de saúde, principalmente nas estratégias sub-regionais. As diferenças entre as metas avaliadas neste estudo sobre a cobertura de realização do exame de Papanicolau destacam a importância da escolha do critério para estabelecimento de metas para avaliação da cobertura. Sugere-se que novos estudos e políticas de saúde considerem critérios populacionais para avaliação das metas. Acredita-se que os resultados apresentados poderão auxiliar na identificação e correção de possíveis falhas do programa de prevenção do CCU, além de contribuir para a elaboração de novas estratégias e melhorias das ações preventivas e diagnósticas.

\section{REFERÊNCIAS}

1. World Health Organization. WHO guidance note: comprehensive cervical cancer prevention and control: a healthier future for girls and women [Internet]. Genebra: WHO; 2013 [citado em 2014 ago 17]. Disponível em: http://www.who.int/immunization/hpv/learn/comprehensive_cervical_ cancer_who_2013.pdf.

2. Brasil. Ministério da Saúde. Instituto Nacional de Câncer. Rede câncer: câncer do colo do útero [Internet]. Rio de Janeiro: INCA; 2014 [citado em 2014 ago 16]. Disponível em: http://www2.inca.gov.br/wps/wcm/connect/ cancercoloutero/site/home/historico_programa/historico.

3. Barroso MF, Gomes KRO, Andrade JX. Frequência de colpocitologia oncótica em jovens com antecedentes obstétricos em Teresina, Piauí, Brasil. Rev Panam Salud Publica. 2011;29(3):162-8. PMid:21484015.

4. Brasil. Ministério da Saúde. Instituto Nacional de Câncer. Estimativa 2012: incidência de câncer no Brasil. Rio de Janeiro: Inca; 2011.

5. Gustafsson L, Pontén J, Zack M, Adami HO, Adami H-O. International incidence rates of invasive cervical cancer after introduction of cytological screening. Cancer Causes Control. 1997;8(5):755-63. http://dx.doi. org/10.1023/A:1018435522475. PMid:9328198.

6. Brasil. Ministério da Saúde. Instituto Nacional de Câncer. Diretrizes brasileiras para o rastreamento do câncer do colo do útero. Rio de Janeiro: Inca; 2011.

7. Brasil. Ministério da Saúde. Instituto Nacional do Câncer. Normas e Recomendações do INCA: prevenção do câncer do colo do útero. Rev Bras Cancerol. 2003;49(4):205.

8. Brasil. Ministério da Saúde. Instituto Nacional de Câncer. Falando sobre câncer do colo do útero. Rio de Janeiro: Inca; 2002.

9. Brasil. Ministério da Saúde. Instituto Nacional de Câncer. Programa nacional de controle do câncer do colo do útero e programa nacional de controle do câncer de mama. Rio de Janeiro: Inca; 2010

10. Brasil. Ministério da Saúde. Instituto Nacional de Câncer. Sistema de informação do controle do câncer de mama (SISMAMA) e do câncer do colo do útero (SISCOLO): manual gerencial. Rio de Janeiro: Inca; 2011.

11. Bicalho SM, Aleixo JLMO. Programa "Viva Mulher": programa nacional de controle do câncer do colo uterino e mama. Rev Min Saude Publica. 2002;1(1):1-16.

12. Instituto Nacional do Câncer. Periodicidade de realização do exame preventivo do câncer do colo do útero. Rev Bras Cancerol. 2002;48(1):13-5.

13. Alves CMM, Guerra MR, Bastos RR. Tendência de mortalidade por câncer de colo do útero para Estado de Minas Gerais, Brasil, 1980-2005. Cad Saude Publica. 2009;25(8):1693-700. http://dx.doi.org/10.1590/S0102311X2009000800005. PMid:19649410.
14. Instituto Brasileiro de Geografia e Estatística. Estimativas populacionais por ano [Internet]. Rio de Janeiro: IBGE; 2014 [citado em $2014 \mathrm{fev} 10$ ]. Disponível em: http://www.ibge.gov.br/home/estatistica/populacao/ estimativa2013/default.shtm.

15. Agência Nacional de Saúde Suplementar. Acesso à informação [Internet] [citado em 2014 jan 15]. Disponível em: http://www.ans.gov.br/acesso-ainformacao.

16. Murata IMH, Grabrielloni MC, Schirmer J. Cobertura do Papanicolaou em mulheres de 25 a 59 anos de Maringá - PR, Brasil. Rev Bras Cancerol. 2012;58(3):409-15.

17. Silva PV, Araújo A, Araújo MRN. Análise da cobertura do exame citopatológico do colo do útero no município de Doresópolis-MG. Rev Enferm Cent-Oeste Min. 2011;1(2):154-63.

18. Secretaria de Estado da Saúde de Minas Gerais. Acordo de Resultados [Internet]. Belo Horizonte: SES-MG [citado em 2015 ago 24]. Disponível em: http://acordoderesultados.saude.mg.gov.br/arquivos/GABINETE.pdf.

19. StataCorp LP. Stata statistical software: release 13 [Software]. College Station: StataCorp LP; 2013.

20. Brasil. Ministério da Saúde. Departamento de Informática do SUS [Internet] [citado em 2014 jan 12]. Disponível em: http://datasus.saude.gov.br/.

21. Viegas SMF, Penna CMM. O vínculo como diretriz para a construção da integralidade na Estratégia Saúde da Família. Rev RENE. 2012;13(2):37585 .

22. Santos AM, Assis MMA, Rodrigues AAO, Nascimento MAA, Jorge MSB. Linhas de tensões no processo de acolhimento das equipes de saúde bucal do programa saúde da família: o caso de Alagoinhas, Bahia, Brasil. Cad Saude Publica. 2007;23(1):75-85. http://dx.doi.org/10.1590/S0102311X2007000100009. PMid:17187106.

23. Andrade SSC, Silva FMC, Silva MSS, Oliveira SHS, Leite KNS, Sousa MJ. Compreensão de usuárias de uma unidade de saúde da família sobre o exame Papanicolaou. Cien Saude Colet. 2013;18(8):2301-10. http://dx.doi. org/10.1590/S1413-81232013000800014. PMid:23896912.

24. Diógenes MAR, Jorge RJB, Sampaio LRL, Mendonça FAC, Sampaio LL. Barreiras à realização periódica do Papanicolaou: estudo com mulheres de uma cidade do Nordeste do Brasil. Rev APS. 2011;14(1):12-8.

25. Lima-Costa MF, Turci MA, Macinko J. Estratégia saúde da família em comparação a outras fontes de atenção: indicadores de uso e qualidade dos serviços de saúde em Belo Horizonte, Minas Gerais, Brasil. Cad Saude Publica. 2013;29(7):1370-80. http://dx.doi.org/10.1590/S0102-311X2013000700011. PMid:23843004.

26. Santos MS, Macêdo APN, Leite MAG. Percepção de usuárias de uma uniade de saúde da família acerca da prevenção do câncer do colo do útero. Rev APS. 2010;13(3):310-9. 
27. Dias MBK, Tomazelli JG, Assis M. Rastreamento do câncer do colo do útero no Brasil: análise de dados do Siscolo no período de 2002 a 2006. Epidemiol Serv Saúde. 2010;19(3):293-306.

28. Silva DSM, Silva AMN, Brito LMO, Gomes SRL, Nascimento MDSB, Chein MBC. Rastreamento do câncer do colo do útero no Estado do
Maranhão, Brasil. Cien Saude Colet. 2014;19(4):1163-70. http://dx.doi. org/10.1590/1413-81232014194.00372013. PMid:24820599.

Recebido em: Maio 06, 2015

Aprovado em: Set. 14, 2015 